\title{
Exact integer linear programming solvers outperform simulated annealing for solving conservation planning problems
}

\author{
Richard Schuster ${ }^{\text {Corresp., } 1,2}{ }^{\text {, Jeffrey } O}$ Hanson ${ }^{3}$, Matt Strimas-Mackey ${ }^{4}$, Joseph R Bennett ${ }^{1}$ \\ 1 Department of biology, Carleton University, Ottawa, ON, Canada \\ 2 Ecosystem Science and Management Program, University of Northern British Columbia, Prince George, Canada \\ 3 School of Biological Sciences, University of Queensland, Brisbane, Australia \\ ${ }^{4}$ Cornell Lab of Ornithology, Cornell University, Ithaca, United States \\ Corresponding Author: Richard Schuster \\ Email address: richard.schuster@glel.carleton.ca
}

The resources available for conserving biodiversity are limited, and so protected areas need to be established in places that will achieve objectives for minimal cost. Two of the main algorithms for solving systematic conservation planning problems are Simulated Annealing (SA) and exact integer linear programming (EILP) solvers. Using a case study in British Columbia, Canada, we compare the cost-effectiveness and processing times of SA used in Marxan versus EILP using both commercial and open-source algorithms. Plans for expanding protected area systems based on EILP algorithms were 12 to $30 \%$ cheaper than plans using SA, due to EILP's ability to find optimal solutions as opposed to approximations. The best EILP solver we examined was on average 1071 times faster than the SA algorithm tested. The performance advantages of EILP solvers were also observed when we aimed for spatially compact solutions by including a boundary penalty. One practical advantage of using EILP over SA is that the analysis does not require calibration, saving even more time. Given the performance of EILP solvers, they can be used to generate conservation plans in real-time during stakeholder meetings and can facilitate rapid sensitivity analysis, and contribute to a more transparent, inclusive, and defensible decision-making process. 
1 Title: Exact integer linear programming solvers outperform simulated annealing for solving

2 conservation planning problems

3

4 Authors: Richard Schuster ${ }^{\mathrm{a}, \mathrm{b},{ }^{*}, \text { Jeffrey O. Hanson }}{ }^{\mathrm{c}}$, Matt Strimas-Mackey ${ }^{\mathrm{d}}$, Joseph R. Bennett ${ }^{\mathrm{a}}$ 5

$6 \quad{ }^{a}$ Department of Biology, 1125 Colonel By Drive, Carleton University, Ottawa ON, K1S 5B6

7 Canada.

8 b Ecosystem Science and Management Program, 3333 University Way, University of Northern

9 British Columbia, Prince George BC, V2N 4 Z9 Canada.

$10{ }^{\mathrm{c}}$ School of Biological Sciences, The University of Queensland, Brisbane, QLD 4072, Australia

$11{ }^{\mathrm{d}}$ Cornell Lab of Ornithology, Cornell University, Ithaca, NY 14850 USA.

12

13 *Corresponding author: Department of Biology, 1125 Colonel By Drive, Carleton University,

14 Ottawa ON, K1S 5B6 Canada. Email: richard.schuster@glel.carleton.ca, Phone: +1 250631

15 8324, ORCID: 0000-0003-3191-7869 


\section{Abstract}

17 The resources available for conserving biodiversity are limited, and so protected areas need to be

18 established in places that will achieve objectives for minimal cost. Two of the main algorithms

19 for solving systematic conservation planning problems are Simulated Annealing (SA) and exact

20 integer linear programming (EILP) solvers. Using a case study in British Columbia, Canada, we

21 compare the cost-effectiveness and processing times of SA used in Marxan versus EILP using

22 both commercial and open-source algorithms. Plans for expanding protected area systems based

23 on EILP algorithms were 12 to $30 \%$ cheaper than plans using SA, due to EILP's ability to find

24 optimal solutions as opposed to approximations. The best EILP solver we examined was on

25 average 1071 times faster than the SA algorithm tested. The performance advantages of EILP

26 solvers were also observed when we aimed for spatially compact solutions by including a

27 boundary penalty. One practical advantage of using EILP over SA is that the analysis does not

28 require calibration, saving even more time. Given the performance of EILP solvers, they can be

29 used to generate conservation plans in real-time during stakeholder meetings and can facilitate

30 rapid sensitivity analysis, and contribute to a more transparent, inclusive, and defensible

31 decision-making process. 


\section{Introduction}

33

Area-based systematic conservation planning aims to provide a rigorous, repeatable, and structured approach for designing new protected areas that efficiently meet conservation objectives (Margules and Pressey 2000). Historically, spatial conservation decision-making often evaluated parcels opportunistically as they became available for purchase, donation, or under threat (Pressey et al. 1993, Pressey and Bottrill 2008). Although purchasing such areas may improve the status quo, such decisions may not substantially and cost-effectively enhance the long-term persistence of species or communities (Joppa and Pfaff 2009, Venter et al. 2014). Systematic conservation planning, on the other hand, is a multi-step process that involves framing conservation planning problems as optimization problems with clearly defined objectives (e.g. minimize acquisition cost) and constraints (Margules and Pressey 2000). These optimization problems are then solved to obtain candidate reserve designs (termed solutions), which are used to guide protected area acquisitions and land policy (Schwartz et al. 2018). Due to the systematic, evidence-based nature of these tools, they can help contribute to a transparent, inclusive, and more defensible decision-making process (Margules and Pressey 2000).

Today, Marxan is the most widely used systematic conservation planning software, having been used in 184 countries to design marine and terrestrial reserve systems (Ball et al. 2009). Although Marxan supports several algorithms for solving conservation planning problems, most conservation planning exercises use its implementation of simulated annealing (SA), an iterative, stochastic metaheuristic algorithm for approximating global optima of complex functions (Kirkpatrick et al. 1983). By conducting thousands of simulations to determine the impact of different candidate solutions, Marxan aims to generate solutions that are near-optimal. One of the reasons why Marxan uses SA instead of exact integer linear 
55 programming (EILP) solvers, is that EILP solvers were historically not well suited to solve

56 problems with nonlinear constraints and penalties, such as problems trying to create spatially

57 compact or connected solutions (i.e. compactness and connectivity goals) and generally took

58 considerably longer than SA to solve problems (Sarkar et al. 2006, Haight and Snyder 2009).

59 However, the SA approach provides no guarantee on solution quality, and conservation scientists

60 and practitioners have no way of knowing how close to optimal their solutions are. In this case,

61 "optimal" refers to the configuration of protected areas that delivers the desired benefits and the

62 lowest cost. The discussion about the relative merits of linear programming versus heuristics

63 such as SA in conservation planning spans more than two decades (Cocks and Baird 1989,

64 Underhill 1994, Church et al. 1996, Rodrigues and Gaston 2002, Önal 2004), but the EILP

65 shortcomings mentioned above have largely been overcome in recent years (Beyer et al. 2016).

In a recent simulation study, Beyer et al. (2016) found that Marxan with simulated

67 annealing can deliver solutions that are orders of magnitude below optimality. They compared

68 Marxan to exact integer linear programming (EILP) (Wolsey and Nemhauser 1999), which

69 minimizes or maximizes an objective function (a mathematical equation describing the

70 relationship between actions and outcomes) subject to a set of constraints and conditional on the

71 decision variables (the variables corresponding to the selection of actions to implement) being

72 integers (Beyer et al. 2016). Unlike metaheuristic methods such as SA, prioritization using EILP

73 will find the optimal solution or can be instructed to return solutions within a defined level of

74 suboptimality. Some have argued that EILP algorithms are well-suited for solving conservation

75 planning problems (Cocks and Baird 1989, Underhill 1994, Rodrigues and Gaston 2002), but

76 until recent advances in computational capacity and algorithms, it has been impossible to solve 
77 the Marxan-like systematic conservation planning problems with EILP for large problems

78 (Haight and Snyder 2009, Beyer et al. 2016).

Here we compare exact integer linear programming solvers with simulated annealing as used in Marxan, for solving minimum set systematic conservation planning problems (Rodrigues et al. 2000) using real-world data from Western North America. The goal of solving the minimum set problem is to find the places that maximize biodiversity, while minimizing reserve cost. We found that EILP generated high quality solutions 1,000 times faster than simulated annealing that could save over $\$ 100$ million (or 13\%) for realistic conservation scenarios when compared to solutions obtained from simulated annealing. These results also hold true for problems aiming for spatially compact solutions. Our findings open up new possibilities for

87 scenario generation to quickly explore and compare different conservation prioritization 88 scenarios in real-time.

\section{Material and Methods}

Study area

We focused on a $27,250 \mathrm{~km}^{2}$ portion of the Georgia Basin, Puget Trough and Willamette Valley of the Pacific Northwest region spanning the US and Canada, corresponding to the

94 climate envelope indicative of the Coastal Douglas-fir (CDF) Biogeoclimatic zone in 95 southwestern British Columbia (Meidinger and Pojar 1991) (Supplementary Information Figure $96 \mathrm{~S} 1$ ). Land cover in the region is diverse, with approximately $57 \%$ of the land in forest, $8 \%$ as 97 savanna or grassland, $5 \%$ in cropland, $10 \%$ being urban or built and the rest in wetland, water or 98 barren. 
100

101

102

103

104

105

106

107

108

109

110

111

112

113

114

115

116

117

118

119

120

121

122

Biodiversity data.

We used species distribution models for 72 bird species as our conservation features at a 1-ha grid cell resolution (Supplementary Table 1). The distribution models were based on data from eBird, a citizen-science effort that has produced the largest and most rapidly growing biodiversity database in the world (Hochachka et al. 2012, Sullivan et al. 2014). From the 2013 eBird Reference Dataset (http://ebird.org/ebird/data/download) we used a total of 12,081

checklists in our study area, then filtered these checklists to retain only those from March - June to capture the breeding season, $<1.5$ hours in duration, $<5 \mathrm{~km}$ travelled, and a maximum of 10 visits to a given location to improve model fit. Sampling locations $<100 \mathrm{~m}$ apart were collapsed to one location, yielding 5,470 checklists from 2,160 locations, visited from 1-10 times and 2.53 times on average. The R package unmarked (version 0.9-9; Fiske and Chandler 2011) provided the framework for all species distribution models, which necessarily include two parts:

occupancy and detection (Mackenzie et al. 2002). This form of distribution modelling, also known as occupancy modelling, uses the information from repeat visits to a site to infer estimates of detectability of a species as well as estimates of probability of occurrence. For further details on biodiversity data see (Rodewald et al. 2019).

Property layer and land cost.

We incorporated spatial heterogeneity in land cost (Ando et al. 1998, Polasky et al. 2001, Ferraro 2003, Naidoo et al. 2006) in our plans by using property data and 2012 land value assessments from the Integrated Cadastral Information Society of British Columbia (BC). This process resulted in 193,623 properties for BC which were subsequently used as planning units (Schuster et al. 2014). Property data, including tax assessment land values from Washington 
123 State came from the University of Washington's Washington State Parcel Database

124 (https://depts.washington.edu/wagis/projects/parcels/; Version:

125 StatewideParcels_v2012n_e9.2_r1.3; Date accessed: 2015/04/30), as well as San Juan County

126 Parcel Data with separate signed user agreement. The combined property layer included 1.92

127 million polygons. Property data, including tax assessment land values from Oregon State had to

128 be sourced from individual counties, which included Benton, Clackamas, Columbia, Douglas,

129 Lane, Linn, Marion, Multnomah, Polk, Washington and Yamhill. The combined property layer

130 for Oregon included 605,425 polygons. We converted the polygon cost values to 1 -ha raster cells

131 for consistency with the biodiversity data by calculating area weighted mean values of cost per

132 raster cell. Using tax assessment values as an estimate of conservation cost is an underestimate

133 because tax assessment values are often lower than market value, but estimates of market values

134 over larger areas are rarely available and tax assessments do provide a good general

135 approximation.

136

137 Spatial prioritization

138 We compared EILP and SA for solving the minimum set spatial prioritization problem

139 (Ball et al. 2009). In this formulation, the landscape is divided into a set of discrete planning

140 units. Each planning unit is assigned a financial cost (here we use the assessed land value) and a

141 conservation value for a set of features that we wish to protect (here the occupancy probability

142 for a set of species). We also define representation targets for each species as the amount of

143 habitat we hope to protect for that species. The goal of this prioritization problem is to optimize

144 the trade-off between conservation benefit and financial cost (McIntosh et al. 2017). Achieving

145 this goal involves finding the set of planning units that meets the conservation targets for the 
146 minimum possible cost (i.e. min cost: such that conservation value $\geq$ target). Details on the

147 Marxan problem formulation can be found in Ball et al. (2009) and the EILP formulation in

148 Beyer et al. (2016) and SI Appendix S2. Three key parameters that are important for Marxan

149 analysis, which we also use here are: species penalty factor, number of iterations, and number of

150 restarts (Ardron et al. 2010). Briefly, the species penalty factor is the penalty given to a reserve

151 system for not adequately representing a feature, the number of iterations determines how long

152 the annealing algorithms will run, and the number of restarts determines how many different

153 solutions Marxan will generate (for more details see SI Appendix S1). For all scenarios, we used

$1541 \mathrm{~km}^{2}$ planning units, generated by aggregating the species and cost data to this coarser

155 resolution from the original 1-ha cells. Aggregation was accomplished by taking the sum of cost

156 data and the mean of species data for all 1-ha cells within the larger $1 \mathrm{~km}^{2}$ cells.

EILP solvers (commercial vs open source)

160 are available. All solvers yield optimal solutions to EILP problems, but there are substantial

161 differences in performance (i.e. time taken to solve a problem) and in the size of problems that

162 can be solved (Lin et al. 2017). For the purposes of performance testing we opted for one of the

163 best commercial solvers currently available, Gurobi (Gurobi Optimization Inc. 2017). In a recent

164 benchmark study, Gurobi outperformed other solver packages for more complex formulations

165 and a practical use-case (Luppold et al. 2018). To investigate solver performance of packages

166 that are freely available to everyone, we also tested the open source solver SYMPHONY (Ralphs

167 et al. 2019). Both Gurobi and SYMPHONY can be used from R. For Gurobi we used the R

168 package provided with the software (Gurobi version 8.1-0) and for SYMPHONY the 
169 Rsymphony package (version 0.1-28; Harter et al. 2017). We used the prioritizr R package to

170 solve EILP problems for both Gurobi and SYMPHONY solvers (Hanson et al. 2019).

172 Scenarios investigated

173 We investigated a range of scenarios that were computationally feasible for this study.

174 For both Marxan and prioritzr we created the following range of scenarios: i) vary conservation

175 targets between 10 and $90 \%$ protection of features in $10 \%$ increments ( 9 variations), using ii) 10

$176-72$ features (5 variations) as targets, and iii) with spatial extents of 9,282 planning units, 37,128

177 planning units, and 148,510 planning units (3 variations), resulting in a total of 135 scenarios

178 created (Table 1). For Marxan, we also varied two additional parameters, i) the number of

179 iterations ranged from $10^{4}$ to $10^{8}$ (5 variations) and ii) species penalty factors (SPF) of $1,5,25$,

180 and 125 were explored (4 variations, roughly spanning two orders of magnitude) for a total of

181 2,700 scenarios investigated in Marxan (Table 1). Exploring ranges of values for number of

182 iterations and SPF is recommended for calibration of Marxan to increase its ability to

183 approximate the optimal solution (Ardron et al. 2010). As the processing time for the most

184 complex problem in Marxan (90\% target, 72 features, 148,510 planning units, $10^{8}$ iterations) was

$185>8$ hours, we restricted the full range of scenarios to those mentioned above. The maximum

186 number of planning units we used is within the range of previous studies using Marxan (e.g.

187 Venter et al. 2014; Runge et al. 2016), although using more than 50,000 planning units with SA

188 is discouraged without extensive parameter calibration, as near optimal solutions will be hard to

189 find for problems of that size (Ardron et al. 2010). To allow for a fair contrast between SA and

190 EILP that focuses on algorithmic comparisons and not within SA variation, we focused our

191 results and discussion on the best solution achieved with Marxan across 10 repeat runs. 
As systematic conservation planners often aim for spatially compact solutions to their

193 problems, we also investigated a range of scenarios using a term called boundary length

194 modified (BLM), which is used to improve the clustering and compactness of a solution

195 (McDonnell et al. 2002). We randomly selected a 225 x 225 pixel region of the study area to

196 generate a problem with 50, 625 planning units, the maximum recommended for Marxan. After

197 initial calibration we set the number of features/species to 72, SPF to 25 and number of iterations

198 for Marxan to $10^{8}$. We varied targets between 10 and $90 \%$ protection of features in $10 \%$

199 increments, and used the following BLM values: $0.1 ; 1 ; 10 ; 100 ; 1,000$ for a total of 45

200 scenarios. Both Marxan and prioritzr allow a user to specify BLM values as presented here. For

201 details on the mathematical formulation of the spatial compactness constraint in ILP, please see

202 SI Appendix S2 and (Beyer et al. (2016).

203 All analyses were conducted on a desktop computer with an Intel Core i7-7820X

204 Processor and 128 GB RAM running Ubuntu 18.04 and R v 3.5.3. All data, scripts and full

205 results are available online (https://osf.io/my8pc/) and will be archived in a persistent repository 206 with a DOI pending acceptance of the manuscript.

\section{Results}

EILP algorithms (Gurobi, SYMPHONY) outperformed SA (Marxan) in terms of their

210 ability to find minimal cost solutions across all scenarios that met conservation targets.

211 Summarizing across calibrated Marxan scenarios (number of iterations $>100,000$ and species

212 penalty factor 5 or 25 ), the range of savings ranged from $0.8 \%$ to $52.5 \%$ (median $12.6 \%$, SI

213 Figure S2) when comparing EILP results to the best (cheapest) solution for a Marxan scenario.

214 For example, at the $30 \%$ protection target EILP solvers resulted in solutions that were $\$ 55$ 
215 million cheaper than SA (Figure 1a), because the EILP solvers selected cheaper and fewer

216 parcels in the optimal solution. With these savings an additional 961 ha could be protected

217 (13,897 ha vs 12,936 ha) using an EILP algorithm by raising the representation targets until the

218 cost of the resulting solution matched that of the Marxan solution using SA. In general, SA

219 performed reasonably well at smaller problem sizes, fewer planning units and features and low

220 targets, but as the problem size and complexity increased SA was less consistent in finding good

221 solutions (SI Figure S2). Cost profiles across targets, number of features and number of planning

222 units are shown in SI Figures S3-5.

223

224 The shortest processing times were achieved using the prioritizr package and the

225 commercial solver Gurobi, followed by prioritizr and the open source solver SYMPHONY, and

226 lastly Marxan (Figure 1b). Gurobi had the shortest processing times across all scenarios

227 investigated, SYMPHONY tied with Gurobi in some scenarios and took up to 78 times longer

228 than Gurobi in other scenarios (mean $=14$ times, SI Figure S6), and Marxan took between 1.8

229 and 1995 times longer than Gurobi (mean $=281$ times, SI Figure S7). The longest processing

230 times for Gurobi, SYMPHONY and Marxan for a single scenario were 40 seconds, 31 minutes,

231 and 8 hours respectively. For the most complex problem (i.e. targets $=90 \%, 72$ features; 148,510

232 planning units), Marxan calibration across the 5 number of iterations and 4 species penalty factor

233 values took a total of 5 days 7 hours, compared to 30 seconds using Gurobi and 28 minutes using

234 SYMPHONY. Time profiles across targets, number of features and number of planning units are

235 shown in SI Figures S8-10.

236 EILP algorithms (Gurobi, SYMPHONY) also outperformed SA (Marxan) when using a

237 BLM to achieve more compact solutions. This was true for objective function values (Figure 2a) 
238 as well as for processing times (Figure 2b). Through finding optimal solutions, using EILP

239 resulted in objective function values 5.65 to $149 \%$ (mean 22.7\%) lower than SA values. Gurobi

240 was the fastest solver to find solutions to problems including BLM in 44 of 45 scenarios, in one

241 case SYMPHONY was faster. SYMPHONY outperformed Marxan in 44 of 45 scenarios, and

242 took on average 13.7 times as long as Gurobi to find a solution (range -0.31 to 42.6 ). Marxan

243 was never faster than Gurobi and took on average 104.6 times as long as Gurobi to find a

244 solution (range 3.09 to 190.8). An example of the spatial representation of the solutions for a

$24510 \%$ target is shown in SI Figure S11.

247 Discussion

248 We found that EILP algorithms outperformed SA both in terms of cost-effectiveness and 249 processing times, even when including linearized non-linear problem formulations, when 250 planning for spatially compact solutions. There have been calls for using EILP in solving 251 conservation planning problems in the past (Underhill 1994, Rodrigues and Gaston 2002), but

252 we are now at a point where making this switch is both advisable and computationally feasible, 253 where technical capacity exists. Our study provides a systematic test, using real world data to 254 build on the findings of (Beyer et al. 2016), and shows that their results hold for a realistic case 255 study. We further expanded the scope of testing to include assessed land values in order to give 256 estimates of how much better optimal solution can perform in terms of cost savings, compared to 257 SA solutions. Finally, we showcase that even open source EILP solvers are much faster than SA 258 algorithms as implemented in Marxan, which is very encouraging for non-academic user that 259 would otherwise have to buy Gurobi licenses (Gurobi is free for academic use). The combination 260 of the superior performance findings by both (Beyer et al. 2016) and this study indicates that 
261 EILP approaches should be strongly considered as improvements for minimum set conservation

262 planning problems, currently solved using SA. This improvement is especially important in real

263 world applications as the speed of generating solutions can be advantageous in iterative and

264 dynamic planning processes that usually occur when planning for conservation (Sarkar et al.

265 2006). Given Marxan's flexibility to use optimization methods other than SA, we hope that a

266 future version of Marxan will include EILP solvers.

267 One practical advantage of using EILP over SA is that the analysis does not require

268 parameter calibration. Unlike EILP, parameter calibration is a crucial task in every Marxan/SA

269 project and the species penalty factors, number of SA iterations, and number of SA restarts must

270 be calibrated to improve solution quality (Ardron et al. 2010). This task can be very time

271 consuming, especially for larger problems (e.g. 50,000 planning units). Ideally all possible

272 combinations of parameters should be explored, but this further increases processing time. For

273 instance, exploring three different parameter values would result in 27 different scenarios to

274 explore (i.e. $3 \times 3 \times 3$ ). Although we omitted calibration runs prior to finalizing and presenting

275 results in this study, the parameter calibration step took several days for the most complex

276 problem we investigated in this study. Yet none of this calibration time is necessary using EILP.

277 An added benefit is that the somewhat subjective process of setting values for these three

278 parameters can be eliminated using EILP as well.

279

Recommended practices for Marxan analyses caution against using SA for conservation

280 planning exercises with more than 50,000 planning units (Ardron et al. 2010). Such large-sized

281 problems have occurred in the past and, as increasingly high resolution data become available,

282 may become more common in the future (e.g. Venter et al. 2014; Runge et al. 2016). Unlike SA,

283 EILP/prioritizr can solve problem sizes with more than one million planning units (Hanson 2018, 
284 Schuster et al. 2019). Realistically, as problem sizes grow beyond what was intended for

285 Marxan/SA projects, EILP will run into problems solving very large problems ( $>1$ million

286 planning units) that include non-linear constraints, such as optimizing compactness or

287 connectivity, as those problem formulations need to be linearized for EILP to work. A potential

288 future solution to this issue could be the use of nonlinear integer programming for more

289 problems including non-linear constraints (Grossmann 2002, Lee and Leyffer 2011). Whether

290 EILP would also outperform SA for more complex problem formulations, such as dynamic

291 problems or problems with multiple objectives, still needs to be explored. Potential solutions

292 would be to linearize the problem, or incorporate algorithms like Mixed Integer Quadratically

293 Constrained Programming (Franco et al. 2014).

294 Finally, we argue that another strength of EILP solvers, especially Gurobi, is that they

295 can be used to quickly explore and compare different conservation prioritization scenarios in

296 real-time. This ability could be used to great advantage during stakeholder meetings, to explore

297 various scenarios and undertake rapid sensitivity analysis.

298

299 Conclusion

300 EILP algorithms substantially outperform SA as used in minimum set systematic

301 conservation planning, both in terms of solution cost, as well as in terms of time required to find

302 near optimal or optimal solutions. Using an EILP algorithm, as implemented in the R package

303 prioritizr, has the added benefit that users do not need to worry about or set parameters such as

304 species penalty factors or number of iterations, which significantly reduces the time a user

305 spends on finding suitable values for these parameters. Given the potential EILP is showing for 
306 conservation planning, we recommend users consider adding this modified approach to solving 307 systematic conservation planning problems. 


\section{Acknowledgements}

309 We thank W. Hochachka for providing code fore processing eBird data and three

310 reviewers for insightful comments. All data, scripts and full results are available online

311 (https://osf.io/my8pc/) and will be archived in a persistent repository with a DOI pending

312 acceptance of the manuscript.

313 


\section{References}

315 Ando, A. et al. 1998. Species Distributions, Land Values, and Efficient Conservation. - Science 279: 2126-2128.

Hanson, J. 2018. Conserving evolutionary processes.

344 Hanson, J. et al. 2019. prioritizr: Systematic Conservation Prioritization in R, Version 4.0.2.

345 Harter, R. et al. 2017. Rsymphony: SYMPHONY in R. 
346 Hochachka, W. M. et al. 2012. Data-intensive science applied to broad-scale citizen science. Trends in ecology \& evolution 27: 130-137.

348

349

350

351

352

353

354

355

356

357

358

359

360

361

362

363

364

365

366

367

368

369

370

371

372

373

374

375

376

377

378

Joppa, L. N. and Pfaff, A. 2009. High and far: biases in the location of protected areas. - PloS one 4: e8273.

Kirkpatrick, S. et al. 1983. Optimization by Simulated Annealing. - Science 220: 671-680.

Lee, J. and Leyffer, S. 2011. Mixed Integer Nonlinear Programming. - Springer Science \& Business Media.

Lin, C. Y. et al. 2017. Participant Selection Problem: Relative Performance of Five Optimization Solvers. - Proceedings of the 8th International Conference on Computer Modeling and Simulation: 24-31.

Luppold, A. et al. 2018. Evaluating the performance of solvers for integer-linear programming. in press.

Mackenzie, D. I. et al. 2002. Estimating site occupancy rates when detection probabilities are less than one. - Ecology 83: 2248-2255.

Margules, C. R. and Pressey, R. L. 2000. Systematic conservation planning. - Nature 405: 24353.

McDonnell, M. D. et al. 2002. Mathematical Methods for Spatially Cohesive Reserve Design. Environmental Modeling \& Assessment 7: 107-114.

McIntosh, E. J. et al. 2017. The Impact of Systematic Conservation Planning. - Annual Review of Environment and Resources 42: annurev-environ-102016-060902.

Meidinger, D. and Pojar, J. 1991. Ecosystems of British Columbia. - British Columbia Ministry of Forests.

Naidoo, R. et al. 2006. Integrating economic costs into conservation planning. - Trends in ecology \& evolution 21: 681-7.

Önal, H. 2004. First-best, second-best, and heuristic solutions in conservation reserve site selection. - Biological Conservation 115: 55-62.

Polasky, S. et al. 2001. Selecting Biological Reserves Cost-Effectively: An Application to Terrestrial Vertebrate Conservation in Oregon. - Land Economics 77: 68-78.

Pressey, R. L. and Bottrill, M. C. 2008. Opportunism, Threats, and the Evolution of Systematic Conservation Planning. - Conservation Biology 22: 1340-1345.

Pressey, R. et al. 1993. Beyond opportunism: key principles for systematic reserve selection. Trends in ecology \& evolution 8: 124-128.

Ralphs, T. et al. 2019. coin-or/SYMPHONY: Version 5.6.17. - Zenodo. 
379

380

381

382

383

384

385

386

387

388

389

390

391

392

393

394

395

396

397

398

399

400

401

402

403
Rodewald, A. D. et al. 2019. Tradeoffs in the value of biodiversity feature and cost data in conservation prioritization. - Sci Rep 9: 1-8.

Rodrigues, A. S. L. and Gaston, K. J. 2002. Optimisation in reserve selection procedures-why not? - Biological Conservation 107: 123-129.

Rodrigues, A. S. et al. 2000. Flexibility, efficiency, and accountability: adapting reserve selection algorithms to more complex conservation problems. - Ecography 23: 565-574.

Runge, C. A. et al. 2016. Incorporating dynamic distributions into spatial prioritization (N Roura-Pascual, Ed.). - Diversity and Distributions 22: 332-343.

Sarkar, S. et al. 2006. Biodiversity Conservation Planning Tools: Present Status and Challenges for the Future. - Annual Review of Environment and Resources 31: 123-159.

Schuster, R. et al. 2014. Bird Community Conservation and Carbon Offsets in Western North America. - Plos One in press.

Schuster, R. et al. 2019. Optimizing the conservation of migratory species over their full annual cycle. - Nature Communications 10: 1754.

Schwartz, M. W. et al. 2018. Decision Support Frameworks and Tools for Conservation. Conservation Letters 11: e12385.

Sullivan, B. L. et al. 2014. The eBird enterprise: an integrated approach to development and application of citizen science. - Biological Conservation 169: 31-40.

Underhill, L. G. 1994. Optimal and suboptimal reserve selection algorithms. - Biological Conservation 70: 85-87.

Venter, O. et al. 2014. Targeting Global Protected Area Expansion for Imperiled Biodiversity. PLOS Biology 12: e1001891.

Wolsey, L. A. and Nemhauser, G. L. 1999. Integer and combinatorial optimization. - John Wiley \& Sons. 


\section{Table 1 (on next page)}

Scenarios investigated in our analysis

The total number of scenarios tested for both Gurobi and SYMPHONY are 135. For Marxan analysis, we included calibration steps as well, which brought the total number of scenarios to 2700 for that algorithm. 


\begin{tabular}{llcc} 
Parameter & Value range & variations & Scenarios \\
\hline targets & $10-90 \%$ & 9 & \\
\# features & $10,26,41,56,72$ & 5 & 135 (ILP) \\
\# planning units & $9,282,37,128,148,510$ & 3 & \\
\hline Marxan iterations & $10^{4}, 10^{5}, 10^{6}, 10^{7}, 10^{8}$ & 5 & 2,700 (SA) \\
Marxan SPF & $1,5,25,125$ & 4 & \\
\hline
\end{tabular}




\section{Figure 1}

Solution cost and time comparisons

a) The lines represent costs compared to the Gurobi cost baseline. The numbers on the blue line represent total cost of a solution in million $\$$ and the numbers on the green line represent how much more expensive, again in million \$, the SA/Marxan solution is compared to the ILP solutions. b) Time to solution comparisons between solvers. Marxan parameters used are: 72 features, 37,128 planning units, $10^{7}$ iterations, using mean cost and time, across all Marxan runs that met their target for a given scenario ( $\max =10$ ). Note that in a) gurobi (red) and Rsymphony (blue) yielded optimal solutions for all target values and so their lines are plotted exactly on top of each other. 

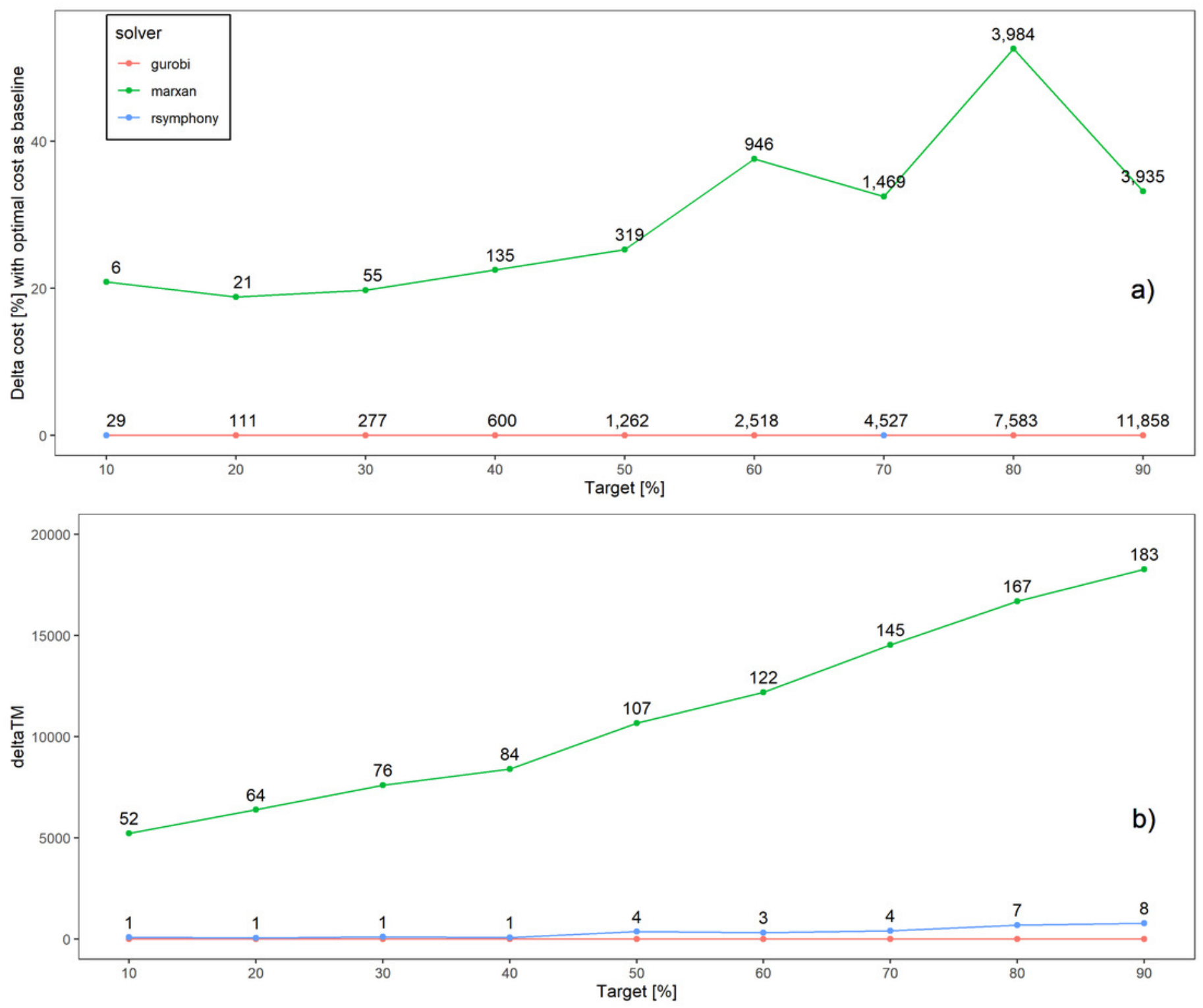


\section{Figure 2}

Objective function value and time comparisons using a boundary penalty to achieve spatially compact solutions

a) Deviation from lowest objective function value for solvers used and over a range of boundary penalty or boundary length modifier values (BLM); zero deviation indicates optimal solution. b) Time to solution comparisons between solvers and across BLM values. Note that in a) gurobi (red) and Rsymphony (blue) yielded optimal solutions for all target values and so their lines are plotted exactly on top of each other. 

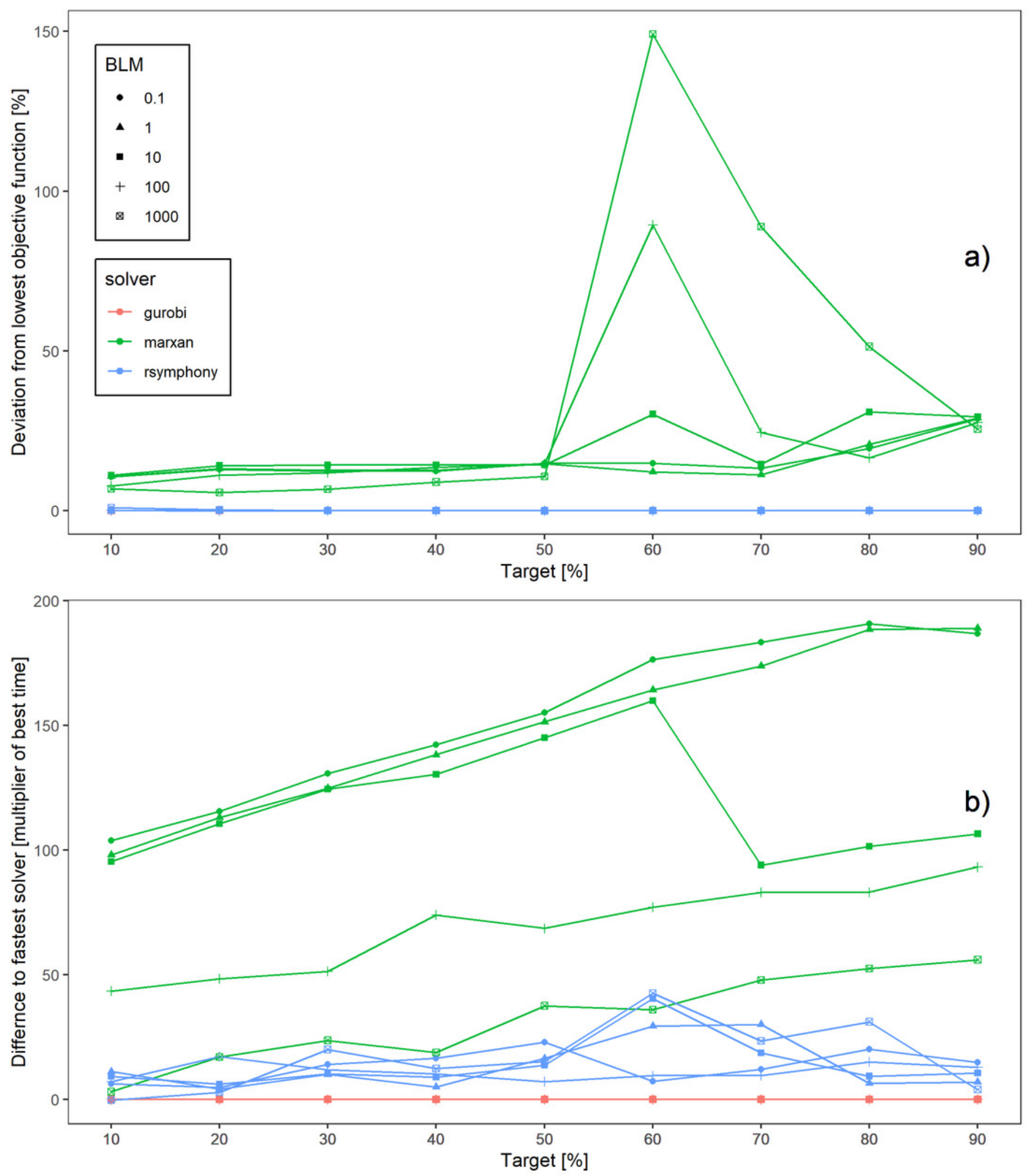